\title{
A Simple Integrated Geometrical Error Model for Laser Tracer
}

\author{
Huixu Song , Hongfang Chen and Zhaoyao Shi \\ Beijing Engineering Research Center of Precision Measurement Technology and Instruments, Beijing University of Technology, \\ Beijing, 100124, China
}

\begin{abstract}
Geometrical errors of 2D gimbal mount axes are studied for laser tracer for the sake of realizing rapid detection for numerical control equipment. The high precision reference sphere, which serves as the reflection unit and is set on the base, avoids the influence on measurement accuracy caused by the movements of rotatory axes, and ensures that laser tracer owns large tracing angle. The nonlinear coupling relationship between the geometrical errors and the measurement accuracy of laser tracer is analyzed; the integrated geometrical error model of 2D gimbal mount axes and laser interferometry system is established; the error map of the measuring system is brought. The simulation results demonstrate that the measurement error of the laser tracer is certain and unique after the value and direction of all the geometrical errors determined. (Key words: Laser tracer; gimbal mount axes; geometrical error model; error map.)
\end{abstract}

\section{Introduction}

The laser tracer holds a high precision reference sphere as the reflection unit whose form error is less than $50 \mathrm{~nm}$, and it can ensure the measurement accuracy by using high precision and high stability laser interferometry system [1-3]. Laser tracer owns the advantage of high precision and high efficiency, however, the geometrical errors of 2D gimbal mount axes are the major factors for the measurement accuracy of laser tracer, therefore it is necessary to detect and calibrate these geometrical errors. Traditional commercial laser tracker and laser tracer were developed on the basis of theodolite and total station. Because of similar shafting structures, the analytical methods for error models of instruments were also analogous. Deumlich analyzed the geometrical errors' sources which could affect the measurement accuracy of theodolite, and gave the relationship between the single geometrical error of rotatory axes and the measurement error of theodolite [4]. K. Lau et al. were the first to give a model of the squareness error for the laser tracker, a model of dead path error for laser interferometry and a model of alignment error for laser beam [5]. B. Muralikrishnan et al. intensively studied the influence of the angular measurement error for the laser tracker on the laser ranging measurement accuracy, and provided a geometrical model for calibrating the measurement error of the laser tracker [6-9]. Hitherto, there was not any research on the geometrical error model for laser tracer.

This paper deeply analyzes the geometrical errors' sources that influence the laser ranging measurement accuracy of laser tracer. In establishing a unified geometrical error model, the nonlinear coupling relationship between the geometrical errors and the laser ranging measurement error is presented, and the error map is drawn then [10-11].

\section{Geometrical error sources of rotatory axes in Laser Tracer}

The schematic diagram of the laser tracer is shown in Fig. $1[12]$.

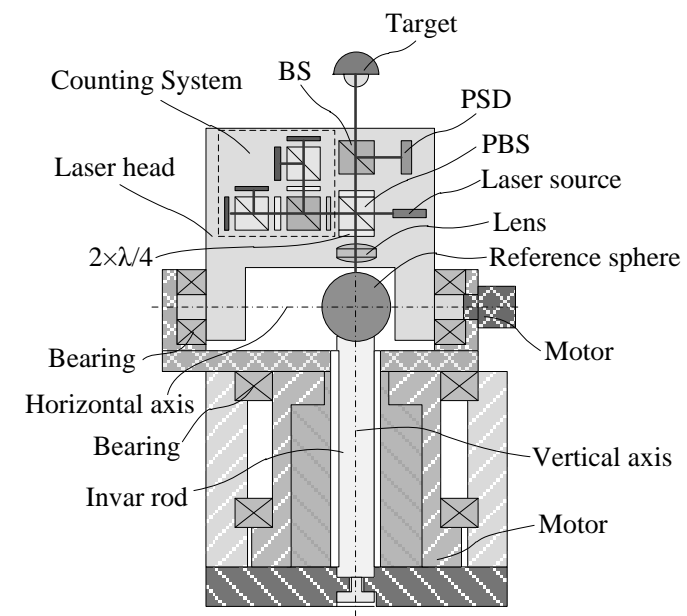

Fig 1. Schematic diagram of laser tracer

In order to clearly identify the characteristics of all the geometrical errors, the geometrical errors are divided into two categories according to the principle of generation - originally geometrical errors and derivatively geometrical errors. The originally geometrical errors include the coaxiality error of horizontal axis (left section and right section), the squareness error of two rotatory axes, the different surface error of two rotatory axes and the cone angle 
error of vertical axis. The derivatively geometrical errors include the eccentricity error of reference sphere and the collimation error of the laser beam.

\subsection{Originally geometrical errors of rotatory axes in Laser Tracer}

As shown in Fig. 2, the coordinate system $O-X Y Z$ is established, with the center of reference sphere $O$ as the origin of coordinate system, the left section of horizontal axis as $\mathrm{X}$, and the vertical axis as Z. $\alpha$ represents the angle between the actual axis and the theoretical axis of horizontal axis; $L_{C}$ represents the offset from the base of laser tracer to horizontal axis; $\theta$ represents the rotation angle of the vertical axis; $\varphi$ represents the rotation angle of the horizontal axis. The geometrical errors' sources of 2D gimbal mount axes can be expressed as follows: $\beta$ squareness error of two rotatory axes; $\gamma$ - cone angle error of vertical axis; $e_{\mathrm{Z}}-$ swing distance of horizontal axis caused by cone angle error of vertical axis; $e_{\mathrm{T}}$ coaxiality error of horizontal axis; $e_{\mathrm{Y}}$ - different surface distance of two rotatory axes, $e_{\mathrm{Y} 1}$ - distance between horizontal axis and the center of reference sphere, $e_{\mathrm{Y} 2}$ distance between vertical axis and the center of reference sphere.

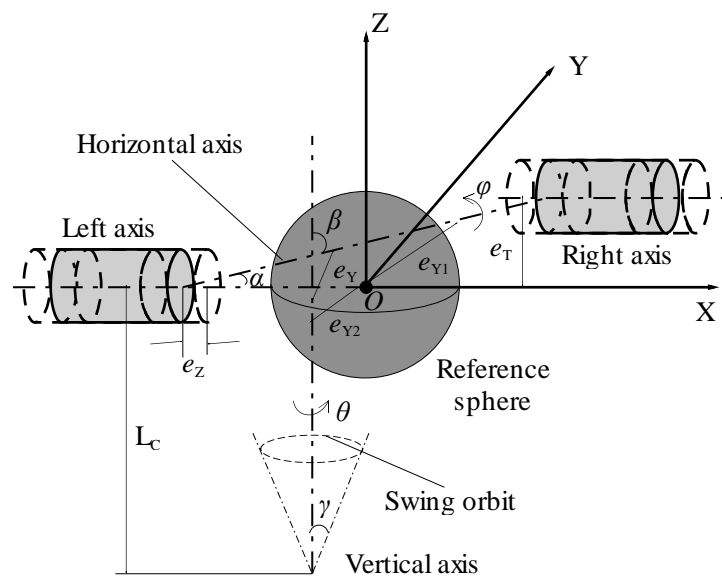

Fig 2. Originally geometrical errors of $2 \mathrm{D}$ gimbal mount axes

\subsubsection{Coaxiality error of horizontal axis}

It is considered that there is only the coaxiality error of horizontal axis $-e_{\mathrm{T}}$, and the intersection of two rotatory axes is located on the axis $Z$. When the vertical axis and the horizontal axis are rotated respectively, the measurement errors, $\Delta_{\mathrm{TV}}$ and $\Delta_{\mathrm{TH}}$, caused by the coaxiality error of horizontal axis are shown as (1) and (2):

$$
\begin{gathered}
\Delta_{\mathrm{TV}}=0 \\
\Delta_{\mathrm{TH}}=\frac{e_{\mathrm{T}} \cdot \cos \alpha}{4} \cdot\left[\sin \left(\varphi-\frac{\pi}{2}\right)+1\right]
\end{gathered}
$$

\subsubsection{Squareness error of two rotatory axes}

It is considered that there is only squareness error of two rotatory axes - $\beta$, and this error does not produce any eccentricity between the two rotatory axes and the reference sphere. Laser tracer is based on the principle of multi-lateration and does not need the angles of two rotatory axes to determine the location of the target. Therefore, the measurement error, $\Delta_{\mathrm{C}}$, caused by the squareness error of two rotatory axes is zero.

\subsubsection{Different surface error of two rotatory axes}

It is considered that there is only the different surface error of two rotatory axes $-e_{\mathrm{Y}}$, and $e_{\mathrm{Y} 1}$ and $e_{\mathrm{Y} 2}$ are both located on the axis Y. When the vertical axis and the horizontal axis are rotated respectively, the measurement errors, $\Delta_{\mathrm{YV}}$ and $\Delta_{\mathrm{YH}}$, caused by the different surface error of two rotatory axes are shown as (3) and (4):

$$
\begin{aligned}
& \Delta_{\mathrm{YV}}=\frac{e_{\mathrm{Y} 2}}{2} \cdot[\cos (\theta-\pi)-1] \\
& \Delta_{\mathrm{YH}}=\frac{e_{\mathrm{Y} 1}}{2} \cdot\left[\sin \left(\varphi-\frac{\pi}{2}\right)+1\right]
\end{aligned}
$$

\subsubsection{Cone angle error of vertical axis}

It is considered that there is only the cone angle error of the vertical axis - $\gamma$, and this error produces a certain eccentricity between the two rotatory axes and the reference sphere, namely $e_{\mathrm{Z}}=\mathrm{L}_{\mathrm{C}} \times \tan \gamma$. Under the action of this eccentricity, the laser head is still rotated around the reference sphere at a constant radius. Therefore, measurement errors, $\Delta_{\mathrm{ZV}}$ and $\Delta_{\mathrm{ZH}}$, caused by the cone angle error of vertical axis are both zero, when the vertical axis and the horizontal axis are rotated respectively.

\subsection{Derivatively geometrical errors of rotatory axes in Laser Tracer}

The analysis above shows that the geometrical errors of 2D gimbal mount axes for laser tracer not only can affect the intersection state of two rotatory axes but also can affect the relationship between two rotatory axes and reference sphere. Therefore, the originally geometrical errors will result in the eccentricity error of reference sphere and the collimation error of laser beam, shown as Fig. 3.

When there are only the eccentricity error of reference sphere and the collimation error of the laser beam in laser tracer, the center of reference sphere does not overlap the origin of coordinate system, and the laser beam does not pass through the center of reference sphere. The coordinate system $O-X Y Z$ is established, with the horizontal axis as $\mathrm{Y}$, the vertical axis as $\mathrm{Z}$, and the intersection $O$ of two rotatory axes as the origin of coordinate system. Point $b_{1}$ represents the original position of the target; point $s_{1}$ represents the position of laser spot on the surface of reference sphere when the 
target is at the original position; point $b_{0}$ represents the intersection between laser beam and plane YOZ; point $b_{1}{ }^{\prime}$ represents the final position of the target after being rotated around vertical axis as $\theta$ and horizontal axis as $\varphi$ with the radius of $L$; point $s_{1}{ }^{\prime}$ represents the position of laser spot on the surface of reference sphere when the target is at the final position; point $b_{0}{ }^{\prime}$ represents the final position of $b_{0}$ after being rotated around the two rotatory axes. The laser beam reflected by reference sphere does not backtrack into counting system, due to the deviation between the laser beam and the center of reference sphere. Change in the direction of reflected beam results in the change in optical path of measuring beam. Influenced by the refraction of optical elements, it is relatively complex to calculate the optical path difference between measuring beam and reference beam. Therefore, we study the characteristics of the collimation error of the laser beam first.

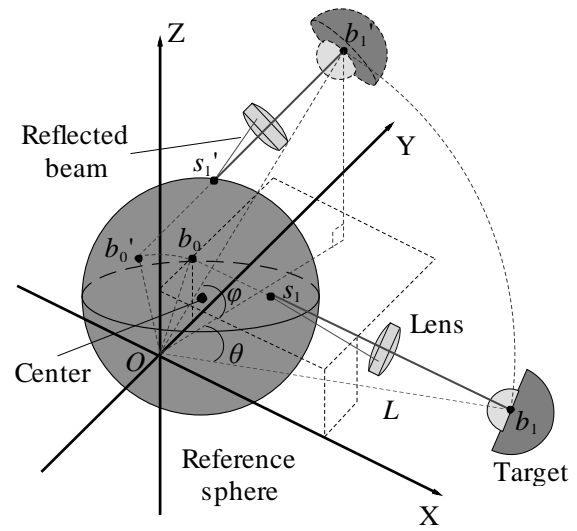

Fig 3. Derivatively geometrical errors of 2D gimbal mount axes

The analysis of the collimation error of the laser beam is shown in Fig. 4. The position of red dashed line represents the theoretical positions of the laser beam and optical elements without the collimation error of the laser beam. The position of blue dashed line represents the theoretical positions of the laser beam and optical elements with the collimation error of the laser beam. The position of purple line represents the actual positions of the laser beam and optical elements when the rotatory axes are controlled by the signals of PSD. $l_{1}$ represents the distance between the target and the center of PBS; $l_{2}$ represents the distance between the center of lens and the center of $\mathrm{PBS} ; l_{3}$ represents the distance between the center of counting system and the center of PBS; $f$ represents the focal length of lens; $r$ represents the radius of reference sphere; $\delta$ represents the collimation error of laser beam; $\omega$ represents the angle of incident beam and reflected beam; $\gamma$ represents the angle of transmitted beam and the optical axis of lens; point $P_{\mathrm{A}}$ to point $P_{\mathrm{I}}$ represent the intersections between laser beam and optical elements.

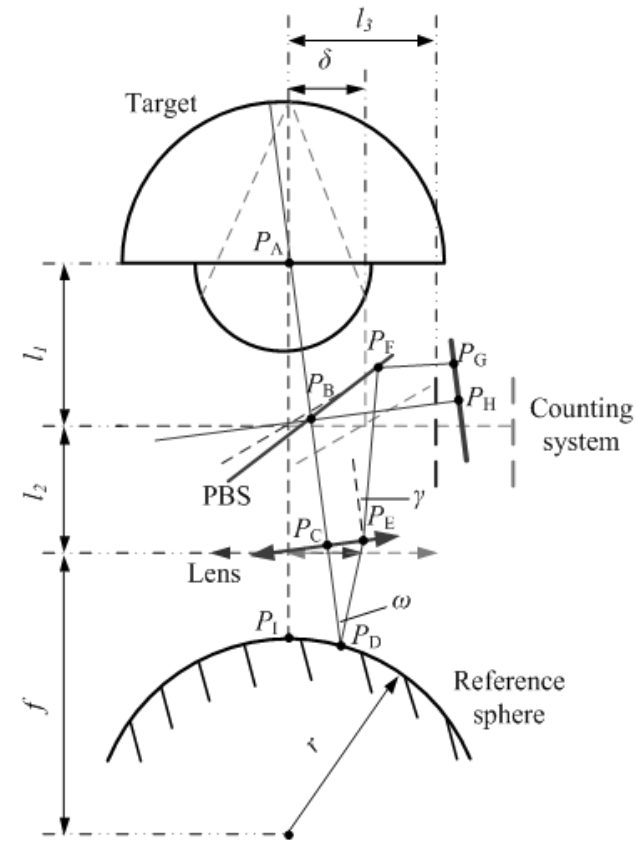

Fig 4. Collimation error of laser beam

When there is the collimation error of the laser beam, the optical path difference between measuring beam and reference beam is shown (see (5)). However, when there is no collimation error of the laser beam, the optical path difference between measuring beam and reference beam is shown (see (6)).

$$
\begin{gathered}
\Delta_{1}=l_{P_{B} P_{A}}+l_{P_{A} P_{D}}+l_{P_{D} P_{E}}+l_{P_{E} P_{F}}+l_{P_{F} P_{G}}-l_{P_{B} P_{H}} \\
\Delta_{2}=2\left(l_{1}+l_{2}+f-r\right)
\end{gathered}
$$

Therefore, the measurement error of the laser ranging caused by the collimation error of the laser beam is given as (7):

$$
\begin{gathered}
\Delta_{\mathrm{D}}=2 \sqrt{\left(l_{1}+l_{2}+f\right)^{2}-\delta^{2}}-\sqrt{r^{2}-\delta^{2}}-\left(l_{2}+f\right)+ \\
\frac{f-\sqrt{r^{2}-\delta^{2}}}{\cos \omega}+\frac{l_{2}+l_{3}}{\cos \gamma}-l_{3}-2\left(l_{1}+l_{2}+f-r\right)
\end{gathered}
$$

Laser ranging measurement error caused by the collimation error of the laser beam is less than $0.1 \mu \mathrm{m}$ if the collimation error is within $\pm 10 \mu \mathrm{m}$ [13-14]. In order to simplify the analysis process, the situation that the incident beam does not overlap the reflected beam can be ignored.

Actually, during the rotation of two rotatory axes, the laser ranging measurement error is coupled nonlinearly by all the geometrical errors of $2 \mathrm{D}$ gimbal mount axes in laser tracer. Therefore, it is necessary to make a comprehensive analysis about all the geometrical errors and calculate the laser ranging measurement error in a unified coordinate system, in order to realize error compensation. 


\section{Integrated model of geometrical errors of axes}

Integrated model of geometrical errors of axes is set as Fig. 5. According to the effect of each geometrical error, the coaxiality error of the horizontal axis is equivalent to the eccentricity error of reference sphere and the squareness error of two rotatory axes. The cone angle error of the vertical axis is equivalent to the collimation error of the laser beam. The coordinate system $O-\mathrm{XYZ}$ is established, with the vertical axis (V) as $\mathrm{Z}$. Axis $\mathrm{X}$ is perpendicular to both the vertical axis and horizontal axis $(\mathrm{H})$ simultaneously. $O_{1}\left(c_{\mathrm{x}}, c_{\mathrm{y}}, c_{\mathrm{z}}\right)$ represents the center of reference sphere, namely the eccentricity error of reference sphere. $b_{0}\left(0, b_{y}, b_{z}\right)$ represents the intersection between the laser beam and plane YOZ, and $\zeta$ represents the angle between the laser beam and plane XOY, which both belong to the collimation error of the laser beam. $\varepsilon$ represents the angle between the horizontal axis and plane XOY, namely the squareness error of two rotatory axes. $e_{x}$ represents the distance between the horizontal axis and plane YOZ, namely the different surface error of two rotatory axes. $L$ is the initial distance between the target and the origin of coordinate system $O$-XYZ, namely constant radius. Point $b$ is the projection of point $b_{1}$ and is on the same plane with point $b_{0} . R$ is the distance between the origin $O$ and point $b . r$ represents the radius of reference sphere. Point $h_{0}$ represents the initial intersection between the horizontal axis and axis $\mathrm{X}$.

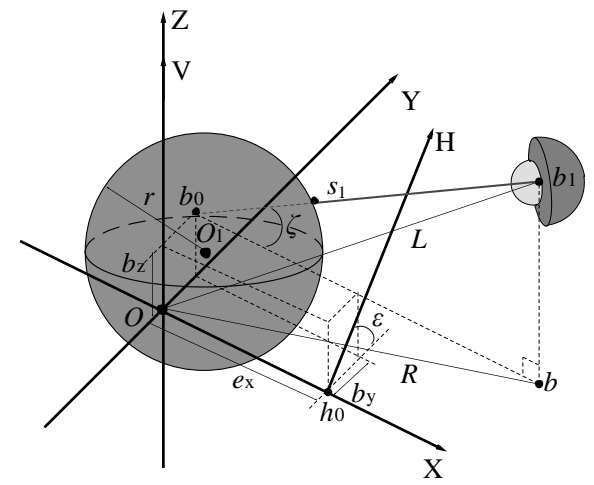

Fig 5. Integrated geometrical error model

The initial intersection $h_{0}$ and direction vector $\mathbf{n}_{\mathbf{H}}$ are given as (8) and (9):

$$
\begin{gathered}
\mathbf{h}_{\mathbf{0}}=\left[\begin{array}{lll}
e_{\mathrm{x}} & 0 & 0
\end{array}\right]^{T} \\
\mathbf{n}_{\mathbf{H} 0}=\left[\begin{array}{ccc}
1 & 0 & 0 \\
0 & \cos \varepsilon & -\sin \varepsilon \\
0 & \sin \varepsilon & \cos \varepsilon
\end{array}\right]\left[\begin{array}{l}
0 \\
1 \\
0
\end{array}\right] \approx\left[\begin{array}{l}
0 \\
1 \\
\varepsilon
\end{array}\right]
\end{gathered}
$$

Point $b_{0}, b_{1}$ and direction vector $\mathbf{n}_{\mathbf{B} 0}$ are given as (10) - (12):

$$
\mathbf{b}_{\mathbf{0}}=\left[\begin{array}{lll}
0 & b_{\mathrm{y}} & b_{\mathrm{z}}
\end{array}\right]^{T}
$$

$$
\begin{aligned}
\mathbf{b}_{1} & =\left[\begin{array}{c}
\sqrt{R^{2}-b_{\mathrm{y}}^{2}-b_{\mathrm{z}}^{2}}-e_{\mathrm{x}} \\
b_{\mathrm{y}} \\
b_{\mathrm{z}}-\xi \sqrt{R^{2}-b_{\mathrm{y}}^{2}-b_{\mathrm{z}}^{2}}
\end{array}\right] \\
\mathbf{n}_{\mathbf{B} 0} & =\left[\begin{array}{ccc}
\cos \xi & 0 & \sin \xi \\
0 & 1 & 0 \\
-\sin \xi & 0 & \cos \xi
\end{array}\right]\left[\begin{array}{l}
1 \\
0 \\
0
\end{array}\right] \approx\left[\begin{array}{c}
1 \\
0 \\
-\xi
\end{array}\right]
\end{aligned}
$$

Point $b_{0}(\theta, \varphi), b_{1}(\theta, \varphi)$ and direction vector $\mathbf{n}_{\mathbf{B}}(\theta, \varphi)$ has changed after rotation, and are given as (13) - (15):

$$
\begin{aligned}
& \mathbf{b}_{\mathbf{0}}(\theta, \varphi)=\left[\begin{array}{lll}
x_{\mathrm{b} 0} & y_{\mathrm{b} 0} & z_{\mathrm{b} 0}
\end{array}\right]=R_{\mathrm{z}}(\theta) \mathbf{h}_{\mathbf{0}} \\
& +R_{\mathrm{z}}(\theta) R_{\mathrm{x}}(\varepsilon) R_{\mathrm{y}}(\varphi) R_{\mathrm{x}}(-\varepsilon)\left[\mathbf{b}_{\mathbf{0}}-\mathbf{h}_{\mathbf{0}}\right] \\
& \mathbf{b}_{\mathbf{1}}(\theta, \varphi)=\left[\begin{array}{lll}
x_{\mathrm{b} 1} & y_{\mathrm{b} 1} & z_{\mathrm{b} 1}
\end{array}\right]=R_{\mathrm{z}}(\theta) \mathbf{h}_{\mathbf{0}} \\
& +R_{\mathrm{z}}(\theta) R_{\mathrm{x}}(\varepsilon) R_{\mathrm{y}}(\varphi) R_{\mathrm{x}}(-\varepsilon)\left[\mathbf{b}_{\mathbf{1}}-\mathbf{h}_{\mathbf{0}}\right] \\
& \mathbf{n}_{\mathbf{B}}(\theta, \varphi)=\left[\begin{array}{lll}
m & n & p
\end{array}\right]= \\
& R_{\mathrm{z}}(\theta) R_{\mathrm{x}}(\varepsilon) R_{\mathrm{y}}(\varphi) R_{\mathrm{x}}(-\varepsilon) \mathbf{n}_{\mathrm{B} 0}
\end{aligned}
$$

Laser spot $s_{1}(\theta, \varphi)=\left(x_{\mathrm{s} 1}, y_{\mathrm{s} 1}, z_{\mathrm{s} 1}\right)$ can be calculated by the equation of spatial line $b_{0} b_{1}$ and the equation of reference sphere, and is given as (16):

$$
\left\{\begin{array}{c}
\frac{x-x_{\mathrm{b} 0}}{m}=\frac{y-y_{\mathrm{b} 0}}{n}=\frac{z-z_{\mathrm{b} 0}}{p} \\
\left(x-c_{\mathrm{x}}\right)^{2}+\left(y-c_{\mathrm{y}}\right)^{2}+\left(z-c_{\mathrm{z}}\right)^{2}=r^{2}
\end{array}\right.
$$

The distance of $l_{\mathrm{s} 1 \mathrm{~b} 1}(\theta, \varphi)$ between $s_{1}$ and $b_{1}$ is given as (17):

$$
\begin{aligned}
& l_{\mathrm{s} 1 \mathrm{~b} 1}(\theta, \varphi)= \\
& \sqrt{\left(x_{\mathrm{s} 1}-x_{\mathrm{b} 1}\right)^{2}+\left(y_{\mathrm{s} 1}-y_{\mathrm{b} 1}\right)^{2}+\left(z_{\mathrm{s} 1}-z_{\mathrm{b} 1}\right)^{2}}
\end{aligned}
$$

The dead path of laser interferometry system is set as $l_{\text {slbl }}(0,0)$. Therefore, $\Delta l$, the measurement error of the laser tracer, is given as (18):

$$
\Delta l=\frac{l_{\mathrm{slbl}}(\theta, \varphi)-l_{\mathrm{slbl}}(0,0)}{2}
$$

\section{Simulation and analysis}

Firstly, all the geometrical errors of 2D gimbal mount axes are measured. Secondly, the measurement error of laser tracer is computed by a unified coordinate system. Finally, the error map of the measuring system for laser tracer is drawn. The integrated geometrical error model is established in Mathematica, and the artificial data of all the geometrical errors are shown in table 1.

Table 1. Simulation value for geometrical error.

\begin{tabular}{|c|c|c|}
\hline Types of geometrical error & $\begin{array}{c}\text { Linear error } \\
\mu \mathrm{m}\end{array}$ & $\begin{array}{c}\text { Angular } \\
\text { error } \mu \mathrm{rad}\end{array}$ \\
\hline
\end{tabular}




\begin{tabular}{|c|c|c|}
\hline $\begin{array}{c}\text { Eccentricity error of } \\
\text { reference sphere }\end{array}$ & $\begin{array}{c}c_{\mathrm{x}}=5.00, \\
c_{\mathrm{y}}=5.00, \\
c_{\mathrm{z}}=5.00\end{array}$ & - \\
\hline $\begin{array}{c}\text { Collimation error of laser } \\
\text { beam }\end{array}$ & $\begin{array}{c}b_{\mathrm{y}}=5 \sqrt{2}, \\
b_{\mathrm{z}}=5 \sqrt{2}\end{array}$ & $\zeta=-0.10$ \\
\hline $\begin{array}{c}\text { Squareness error between } \\
\text { two rotatory axes }\end{array}$ & - & $\varepsilon=0.10$ \\
\hline $\begin{array}{c}\text { Skew lines error between } \\
\text { two rotatory axes }\end{array}$ & $e_{\mathrm{x}}=15.00$ & - \\
\hline \multicolumn{2}{|c}{} \\
\hline \multicolumn{2}{|c|}{}
\end{tabular}

Through simulation and analysis, the measurement error of laser tracer caused by the geometrical errors of 2D gimbal mount axes is obtained, shown as Fig. 6 .

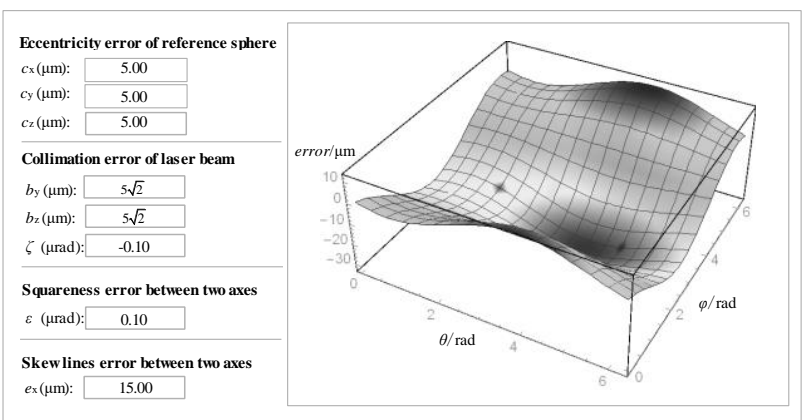

Fig 6. Simulation results for measurement error

Depending on the error map in Fig. 6, the measurement error of laser tracer is the positive maximum $+12.64 \mu \mathrm{m}$, when $\theta=3927.54 \mu \mathrm{rad}$ and $\varphi=$ $6059.66 \mu \mathrm{rad}$. The measurement error of laser tracer is the negative maximum $-32.61 \mu \mathrm{m}$, when $\theta=3927.54$ $\mu \mathrm{rad}$ and $\varphi=2886.82 \mu \mathrm{rad}$. The variation of the measurement error is $45 \mu \mathrm{m}$, in which the contributors for the eccentricity error of reference sphere and the different surface error of two rotatory axes are $17.3 \mu \mathrm{m}$ and $30 \mu \mathrm{m}$ respectively.

For laser tracer, all the geometrical errors of 2D gimbal mount axes must be measured first, and the error map of the measuring system for laser tracer is uploaded to a computer after being drawn by Mathematica. In the process of tracing, the laser ranging measurement value and the compensated data are summed automatically, namely $l+\Delta l$, in which $l$ represents the laser ranging measurement value.

In summary, the different surface error of two rotatory axes and the eccentricity error of reference sphere are major geometrical errors, which need to be strictly controlled. The squareness error of two rotatory axes and the collimation error of the laser beam are minor geometrical errors. Therefore, research on geometrical errors of 2D gimbal mount axes in laser tracer is conducive to improve the measurement accuracy of laser tracer and reduce the processing cost.

\section{Conclusion}

The geometrical errors of 2D gimbal mount axes in laser tracer are studied, and the nonlinear coupling relationship between these geometrical errors and the measurement accuracy is determined. The originally geometrical errors not only can affect the intersection state of two rotatory axes but also can affect the relationship between two rotatory axes and reference sphere. These geometrical errors above will result in the eccentricity error of reference sphere and the collimation error of the laser beam. The integrated geometrical error model is established, and the error map is drawn depending on the relation of spatial geometric transformation. The simulation results demonstrate that the measurement error of laser tracer is a two-variable function of rotation angles of two rotatory axes. This function will not have anything to do with the distance to the target. In other words, the measurement error owns the characteristic of certainty and uniqueness.

\section{References}

1. H. Schwenke, M. Franke, J. Hannaford, H. Kunzmann, CIRP ANN-MANUF TECHN, 54, 475478 (2005)

2. H. Schwenke, R. Schmitt, P. Jatzkowski, C. Warmann, CIRP ANN-MANUF TECHN, 58, 477480 (2008)

3. H.W. Lee, J.R. Chen, S.P. Pan, H.C Liou, P.E. Hsu, APPL SCI, 6, 105 (2016)

4. F. Deumlich, Surveying instruments, 126-146 (1982)

5. K. Lau, R.J. Hocken, W.C. Haight, PRECIS ENG, 8, 3-8 (1986)

6. B. Muralikrishnan, D. Sawyer, C. Blackburn, S. Phillips, B. Borchardt, W.T. Estler, J RES NATL INST STAN, 114, 21-35 (2009)

7. B. Muralikrishnan, S. Phillips, D. Sawyer, PRECIS ENG, 44, 13-28 (2016)

8. B. Muralikrishnan, C. Blackburn, D. Sawyer, S. Phillips, J RES NATL INST STAN, 115, 291-301 (2010)

9. B. Muralikrishnan, M. Shilling, P. Rachakonda, W. Ren, V. Lee, D. Sawyer, J MANUF SYST, 37, 550557 (2015)

10. B. Hughes, A.B. Forbes, A. Lewis, W.J Sun, MEAS SCI TECHNOL, 22, 045103 (2011)

11. B. Hughes, A.B. Forbes, A. Lewis, W.J Sun, Macroscale, (2011)

12. Z.Y. Shi, H.X. Song, H.F. Chen, Y.Q. Sun, MEAS SCI REV, 18, 13-19 (2018)

13. H.X. Song, Z.Y. Shi, H.F. Chen, Y.Q. Sun, Proc. of SPIE, 10621,0C1-0C8 (2018)

14. H.X. Song, Z.Y. Shi, H.F. Chen, Y.Q. Sun, Chinese Journal of Lasers, 45, $0504001 \quad$ (2018) 